\title{
On the construction of the correlation numbers in Minimal Liouville Gravity
}

\author{
Konstantin Aleshkin ${ }^{a, b}$ and Vladimir Belavin ${ }^{c, d, e}$ \\ ${ }^{a}$ L.D. Landau Institute for Theoretical Physics, \\ Akademika Semenova av., 1-A, Chernogolovka, 142432 Moscow region, Russia \\ ${ }^{b}$ International School of Advanced Studies (SISSA), \\ via Bonomea 265, 34136 Trieste, Italy \\ ${ }^{c}$ I.E. Tamm Department of Theoretical Physics, P.N. Lebedev Physical Institute, \\ Leninsky Avenue 53, 119991 Moscow, Russia \\ ${ }^{d}$ Department of Quantum Physics, Institute for Information Transmission Problems, \\ Bolshoy Karetny per. 19, 127994 Moscow, Russia \\ ${ }^{e}$ Moscow Institute of Physics and Technology, \\ Dolgoprudnyi, 141700 Moscow region, Russia \\ E-mail: kkcnst@gmail.com, belavin@lpi.ru
}

ABSTRACT: The computation of the correlation numbers in Minimal Liouville Gravity involves an integration over moduli spaces of complex curves. There are two independent approaches to the calculation: the direct one, based on the CFT methods and Liouville higher equations of motion, and the alternative one, motivated by discrete description of 2D gravity and based on the Douglas string equation. However these two approaches give rise to the results that are not always consistent among themselves. In this paper we explore this problem. We show that in order to reconcile two methods the so-called discrete terms in the operator product expansion in the underlying Liouville theory must be properly taken into account. In this way we propose modified version of the expression for four-point correlation number and find full agreement between direct and alternative approaches. Our result allows to consider correlators without any restrictions on the number of conformal blocks contributing to the matter sector correlation function.

Keywords: 2D Gravity, Conformal Field Models in String Theory, Conformal Field Theory

ARXIV EPRINT: 1610.01558 


\section{Contents}

1 Introduction 1

2 Preliminaries 3

3 Minimal Liouville gravity 5

4 Discrete terms $\quad 7$

5 Generalization of HEM formula $\quad 8$

6 Comparison with Douglas equation approach 11

$\begin{array}{lll}7 & \text { Discussion } & 12\end{array}$

$\begin{array}{ll}\text { A Conformal block decomposition in correlation numbers } & 13\end{array}$

B Proof of the proposition $6.1 \quad 17$

\section{Introduction}

Minimal Liouville Gravity (MLG) represents an interesting solvable model of 2D quantum gravity [1]. The role of the matter in MLG is performed by Minimal Models (MM) of conformal field theory, while the gravitational sector is described by Liouville Field Theory (LFT). The observables are built from the primary fields in both sectors and the definition of the correlation numbers involves an integration over moduli space of n-punctured surface. ${ }^{1}$

The direct approach for evaluating the correlation numbers consists of computing MM and LFT correlation functions, and integrating then their product over the moduli space (see, for example [2]). However, for multi-point correlators this method becomes rather complicated. In [3] so called higher equations of motion (HEM) in LFT [4] were used to reduce the moduli integration in four-point correlation numbers to the boundary terms, however this method has certain restrictions. In what follows we will call "HEM formula" the formula for four-point correlation numbers obtained in [3]. It was derived using an assumption that the correlation function in the matter sector involves a maximal number of conformal blocks in the conformal block decomposition [5].

Another approach to Minimal Liouville Gravity, based on the Douglas string equation ${ }^{2}$ (DSE) and motivated by the Matrix Models, ${ }^{3}$ allows to compute correlation numbers using

\footnotetext{
${ }^{1}$ In this paper we focus on the correlation numbers on a sphere.

${ }^{2}$ We do not discuss another approach to two dimensional gravity - cohomological field theories.

${ }^{3}$ For more details see [6] and references therein.
} 
the specific solution of the Douglas equation [7-9]. The observables in DSE approach have natural scaling properties, which allow to identify them with the observables in MLG [10]. Further research was made in [11] in order to compare the direct and DSE approaches. In [12] three- and four-point correlation numbers in the Lee-Yang series $(2, p)$ of Minimal Models were computed with the help of resonance transformations and the agreement of CFT computation (when it is applicable) and DSE approach computation was shown. However, in [9], where the connection between DSE and Frobenius manifolds was established, it was shown that for $(3, p)$ series of MM it is impossible to obtain full agreement between direct and DSE approaches even on the three-point level. Namely, some of the correlators that should vanish according to MM fusion rules appear to be non zero in the Douglas equation computation. Later, in [13] an alternative description, ${ }^{4}$ also based on the Douglas string equation, was proposed for the Lee-Yang series, that was consistent with the two previous results in the region where HEM formula is applicable but gave different results with the DSE computation based on the resonance transformation in the region where HEM formula cannot be used because of the constraint on the number of conformal blocks.

Thus, we have the following natural questions. The first one is how to correct HEM formula to make it applicable, i.e. consistent with the direct moduli integration, without any restrictions. The second one is which of the two DSE formulae is consistent with the original direct approach if any. The main result of this paper is an answer to both of these questions. Namely, we modify HEM formula for Lee-Yang series so that it is consistent with the direct computation and show that Douglas equation approach as in $[9,12]$ gives the same result.

To generalize HEM formula we notice that in fact it was derived in the case, where the matter sector is represented by the Generalized Minimal Model (GMM) [2]. GMM is a certain modification of the Minimal Models which can have arbitrary central charge $<1$ and fields with arbitrary complex conformal dimensions. There is an explicit formula for the three-point function of this model [2] which coincides with MM three-point function whenever the last one is non-zero. However we find out that in this theory some of the correlation numbers are ill-defined, that becomes clear after analysing analytical structure of GMM 3-point functions and Liouville OPE discrete terms. Taking limits of well-defined correlators with all except one non-degenerate values of the conformal dimensions, we are able to find the exact answer for the genuine Minimal Models correlator.

This consideration allows to modify properly the HEM formula. The corrected formula is in full agreement with all numerical checks and also with the Douglas equation formula, so that it fixes a previous uncertainty for the Lee-Yang series.

The paper is organized as follows. In section 2 we remind some facts from the Minimal Models, GMM and Liouville theory and fix some notations. In section 3 we talk about the Liouville Gravity, construct correlation numbers - the main object of study in this paper and recall HEM formula. In section 4 we discuss discrete terms in Liouville OPE. The main results of the paper are presented in sections 5 and 6. Finally, in the section 7

\footnotetext{
${ }^{4}$ The difference with the previous one is that this approach does not require resonance transformations.
} 
we discuss our results and some further questions. In the appendix we describe the direct approach for computing correlation numbers, present several formulae for convenience and prove some proposition.

\section{Preliminaries}

Here we remind some facts about MLG ingredients, Minimal Models and Liouville Field Theory, and set our notations.

Ordinary and generalized minimal models. Minimal models $\mathcal{M}\left(p^{\prime} / p\right)$ are rational conformal field theories [5]. The fields belong to a sum of a finite number of highest weight representations of Virasoro algebra

$$
\left[L_{n}, L_{m}\right]=(n-m) L_{n+m}+\frac{c}{12}\left(n^{3}-n\right) \delta_{n,-m},
$$

with central charge

$$
c_{M}=1-6 q^{2}
$$

where the parameter $q$ is given by

$$
q=b^{-1}-b, b=\sqrt{p^{\prime} / p} .
$$

In this setting $\left(p^{\prime}-1\right)(p-1)$ primary fields $\Phi_{m, n}(x),{ }^{5}$ correspond to the highest weight vectors with weights $L_{0} \Phi_{m, n}=\Delta_{m, n}^{M} \Phi_{m, n}$. For the conformal dimensions $\Delta_{m, n}^{M}$ it is convenient to introduce parameters

$$
\lambda_{m, n}=\frac{m b+n b^{-1}}{2}, \alpha_{m, n}=\lambda_{m,-n}-q / 2,
$$

so that

$$
\begin{aligned}
\Delta_{m, n}^{M} & =\frac{\left(m b^{-1}-n b\right)^{2}-\left(b^{-1}-b\right)^{2}}{4} \\
& =\frac{\left(m p-n p^{\prime}\right)^{2}-\left(p-p^{\prime}\right)^{2}}{4 p p^{\prime}}=\alpha_{m, n}\left(\alpha_{m, n}-q\right)=q^{2} / 4-\lambda_{m,-n}^{2} .
\end{aligned}
$$

The MM structure constants $\mathbb{C}_{i j}^{k},{ }^{6}$ can be found in [14]. The OPE satisfies the so-called fusion rules which can be symbolically represented as

$$
\Phi_{m_{1}, n_{1}} \Phi_{m_{2}, n_{2}}=\sum_{r, s}\left[\Phi_{r, s}\right]
$$

where $[\Phi]$ denotes the contribution of the primary field $\Phi$ and all its descendants and $\left|m_{1}-m_{2}\right|+1: r: \min \left(m_{1}+m_{2}-1,2 p^{\prime}-m_{1}-m_{2}-1\right),\left|n_{1}-n_{2}\right|+1: s: \min \left(n_{1}+n_{2}-\right.$ $\left.1,2 p-n_{1}-n_{2}-1\right)$. These fusion rules are equivalent to the fusion algebra of integrable $\mathrm{SL}(2)_{p, p^{\prime}}$ representations with $\mathbb{Z} / 2 \mathbb{Z}$ identification $(n, m) \rightarrow\left(p^{\prime}-n, p-m\right)$. The structure constants are zero when these rules are not satisfied.

\footnotetext{
${ }^{5}$ In what follows we omit dependence on $x$ where it is unnecessary.

${ }^{6}$ Here $i, j, k$ run through the pairs $(m, n)$.
} 
For analytic computations in Minimal Liouville Gravity it is instructive to consider Generalized Minimal Models, that is a modification of the ordinary MM. The central charge in GMM can be an arbitrary real number less then one and primary fields of the model can have arbitrary complex dimensions (see for example [2] for discussion). Namely, one introduces primary fields $\Phi_{\alpha}$ with dimension $\Delta_{\alpha}^{M}=\alpha(\alpha-q)$. In particular, one has $\Delta_{m, n}^{M}=\Delta_{\alpha_{m, n}}^{M}$

With this construction more general structure constants $\mathbb{C}^{M}\left(\alpha_{1}, \alpha_{2}, \alpha_{3}\right)$ were calculated in $[2]$

$$
\mathbb{C}^{M}\left(\alpha_{1}, \alpha_{2}, \alpha_{3}\right)=A \Upsilon(\alpha+b-q) \prod_{i} \frac{\Upsilon\left(\alpha-2 \alpha_{i}+b\right)}{\left[\Upsilon\left(2 \alpha_{i}+b\right) \Upsilon\left(2 \alpha_{i}+b-q\right)\right]^{1 / 2}},
$$

where $\alpha=\sum \alpha_{i}$ and the normalization factor

$$
A=\frac{b^{b^{-2}-b^{2}-1}\left[\gamma\left(b^{2}\right) \gamma\left(b^{-2}-1\right)\right]^{1 / 2}}{\Upsilon(b)} .
$$

Here $\gamma(x)=\Gamma(x) / \Gamma(1-x)$ and special function $\Upsilon(x)=\Upsilon_{b}(x)$ is an entire function of complex domain with zeros in $x=-n b^{-1}-m b$ and $(n+1) b^{-1}+(m+1) b$, where $n, m$ are non-negative integers (see for example [15]).

Perhaps, the most important data one can extract from this expression are zeros and poles, which can occur, for example, when the fields are degenerate, that is $\alpha_{i}=\alpha_{m, n}$. In particular, some of the fusion rules arise because of zeros of structure constants corresponding to the degenerate primary fields. We note that not all structure constants, which should vanish according to MM fusion rules, do vanish in GMM. Thus, GMM can not be considered as a direct generalization of Minimal Models.

Liouville field theory. Liouville theory is an irrational CFT with continuous spectrum of primary fields and central charge

$$
c_{L}=1+6 Q^{2}
$$

The primary field $V_{a}$, labeled by complex parameter $a$, has conformal dimension

$$
\Delta_{a}^{L}=a(Q-a) .
$$

Fields with $a=Q / 2+i P$ represent the spectrum of the Liouville CFT. The real parameter $P$ is known as a momentum parameter.

Degenerate fields (i.e. fields, whose Verma module is reducible) are $V_{m, n}=V_{a_{m, n}} \sim$ $V_{Q-a_{m, n}}$, where

$$
a_{m, n}=Q / 2-\lambda_{m, n}
$$

For theses values of the parameter $a$, a singular vector arises on the $m n$-level of the corresponding Verma module [16].

The basic Liouville operator product expansion [15] (for the sake of brevity we write $\Delta=\Delta_{Q / 2+i P}$ and $\left.\Delta_{i}=\Delta_{a_{i}}\right)$

$$
\begin{aligned}
& V_{a_{1}}(x) V_{a_{2}}(0)= \\
& \qquad \int^{\prime} \frac{d P}{4 \pi}(x \bar{x})^{\Delta-\Delta_{1}-\Delta_{2}} \mathbb{C}_{a_{1}, a_{2}}^{Q / 2+i P}\left[V_{Q / 2+i P}(0)\right],
\end{aligned}
$$


where the basic structure constants $\mathbb{C}_{a_{1} a_{2}}^{Q / 2+i P}=\mathbb{C}^{L}\left(a_{1}, a_{2}, Q / 2-i P\right)[17,18]$ (derived from the crossing symmetry in [19]) have the explicit form (here $a$ denotes $a_{1}+a_{2}+a_{3}$ )

$$
\mathbb{C}^{L}\left(a_{1}, a_{2}, a_{3}\right)=\left(\pi \mu \chi\left(b^{2}\right) b^{2-2 b^{2}}\right)^{(Q-a) / b} \frac{\Upsilon_{b}(b)}{\Upsilon_{b}(a-Q)} \prod_{i=1}^{3} \frac{\Upsilon_{b}\left(2 a_{i}\right)}{\Upsilon_{b}\left(a-2 a_{i}\right)},
$$

where $\Upsilon_{b}$ is the same "upsilon" function as the one, which appears in the expression for GMM structure constants (see [18, 20]).

The OPE (2.5) is continuous and involves integration over the "momentum" $P$. The prime on the integral indicates possible discrete terms, which we discuss in more details in section 4. In our computations such extra terms do appear and give an important contribution. We note that because Liouville theory is non-rational and fields of interest do not belong to the spectrum, one cannot use OPE literally as in minimal models but have to apply instead analytic continuation. For example, if one computes 3-point function with the naive OPE, one often gets zero, which is inconsistent with DOZZ formula (2.6) and the 4-point function is inconsistent with conformal bootstrap.

One can notice, that formulae for the central charge and for the conformal dimensions in Liouville theory can be obtained from the ones of GMM by $b \rightarrow i b$. However these two theories are not analytic continuations of each other, because structure constants of these theories can not be obtained as analytic continuations. It becomes clear after noticing that $\Upsilon_{b}(x)$ function have a natural bound of analyticity with respect to parameter $b[2]$.

\section{Minimal Liouville gravity}

In this section we discuss the Minimal Liouville Gravity correlation numbers on a sphere.

In the framework of the so-called DDK approach [21, 22], LG is a tensor product of the conformal matter $(\mathrm{M})$, represented by ordinary or generalized Minimal Models, Liouville theory (L), and the ghost system $(\mathrm{G})$

$$
A_{\mathrm{LG}}=A_{\mathrm{M}}+A_{\mathrm{L}}+A_{\mathrm{G}},
$$

with the interaction via the construction of the physical fields and the relation for the central charge parameters

$$
c_{\mathrm{M}}+c_{\mathrm{L}}+c_{\mathrm{G}}=0 .
$$

The ghost system (see, e.g., [23-25]), consisting of two anticommuting fields $(b, c)$ of spins $(2,-1)$, is the conformal field theory with central charge $c_{\mathrm{G}}=-26$.

Because of the condition (3.1), we should take (generalized) Minimal Models and LFT with the same value of the parameter $b$.

Physical fields and correlation numbers. The physical fields form a space of cohomology classes with respect to the nilpotent BRST charge $\mathbb{Q}$,

$$
\mathbb{Q}=\sum_{m}:\left[L_{m}^{\mathrm{M}+\mathrm{L}}+\frac{1}{2} L_{m}^{\mathrm{g}}\right] c_{-m}:-c_{0}
$$


In general, we deal with the correlators of fields of the type

$$
\mathbb{U}_{a}(z, \bar{z})=\Phi_{a-b}(z, \bar{z}) V_{a}(z, \bar{z}),
$$

where the choice of the parameters ensures that $\mathbb{U}_{a}(z, \bar{z})$ is a $(1,1)$-form, and

$$
\mathbb{W}_{a}(z, \bar{z})=C(z) \bar{C}(\bar{z}) \cdot \mathbb{U}_{a}(z, \bar{z})
$$

which is a scalar field. The parameter $a$ can take generic values.

The $n$-point correlation number on a sphere for these observables [3] is

$$
I_{n}\left(a_{1}, \ldots, a_{n}\right)=\int d^{2} z_{i}\left\langle\prod_{i=4}^{n} \mathbb{U}_{a_{i}}\left(z_{i}\right) \mathbb{W}_{a_{3}}\left(z_{3}\right) \mathbb{W}_{a_{2}}\left(z_{2}\right) \mathbb{W}_{a_{1}}\left(z_{1}\right)\right\rangle .
$$

In what follows we shall be interested in correlators of physical fields in the minimal gravity constructed from the degenerate matter fields $\mathbb{U}_{m, n}=\Phi_{m, n} V_{m,-n}$.

For degenerate matter fields there exists an additional set of so-called ground ring states $[3,26,27]$

$$
\mathbb{O}_{m, n}(z, \bar{z})=\bar{H}_{m, n} H_{m, n} \Phi_{m, n}(z, \bar{z}) V_{m, n}(z, \bar{z}) .
$$

The operators $H_{m, n}$ are composed of Virasoro generators in all three theories and are defined uniquely modulo $\mathbb{Q}$ exact terms. Moreover, if we introduce the logarithmic counterparts of the ground ring states $\mathbb{O}_{m, n}$,

$$
\mathbb{O}_{m, n}^{\prime}=\bar{H}_{m, n} H_{m, n} \Phi_{m, n} V_{m, n}^{\prime},
$$

then we have the following important relation $[3,4]$

$$
\mathbb{U}_{m,-n}=B_{m, n}^{-1} \bar{\partial} \partial \mathbb{O}_{m, n}^{\prime} \bmod \mathbb{Q},
$$

where $B_{m, n}$ are the coefficients arising in the higher equations of motion of LFT [4]. For four points, relation (3.7) allows to reduce the moduli integral in (3.5) to the boundary integrals if one of the fields is degenerate, that is $a_{i}=a_{m,-n}$. It gives

$$
I_{4}\left(a_{m,-n}, a_{2}, a_{3}, a_{4}\right)=\kappa N\left(a_{m,-n}\right)\left(\prod_{i=2}^{4} N\left(a_{i}\right)\right) \Sigma^{(m, n)}\left(a_{2}, a_{3}, a_{4}\right),
$$

where

$$
\Sigma^{(m, n)}(a)=-m n \lambda_{m, n}+\sum_{i=1}^{3} \sum_{r, s}^{(m, n)}\left|\lambda_{i}-\lambda_{r, s}\right|_{\mathrm{Re}},
$$

$\lambda_{i}=Q / 2-a_{i}$ are "momentum parameters" and the fusion set is $(m, n)=\{1-m: 2$ : $m-1,1-n: 2: n-1\}$. The first coefficient in (3.8) is

$$
\kappa=-\left(b^{-2}+1\right) b^{-3}\left(b^{-2}-1\right) Z_{L}, Z_{L}=\left[\pi \mu \gamma\left(b^{2}\right)\right]^{Q / b} \frac{1-b^{2}}{\pi^{3} Q \gamma\left(b^{2}\right) \gamma\left(b^{-2}\right)}
$$

and the "leg" factors are

$$
N(a)=\frac{\pi}{(\pi \mu)^{(a / b)}}\left[\frac{\gamma\left(2 a b-b^{2}\right) \gamma\left(2 a b^{-1}-b^{-2}\right)}{\gamma^{2 a / b-1}\left(b^{2}\right) \gamma\left(2-b^{-2}\right)}\right]^{1 / 2} .
$$


The expression (3.8) was derived under the assumption that the number of conformal blocks in the expansion of the matter sector correlation function is maximally possible, i.e. the number of conformal blocks $=m n$. We discuss this point in more details in sections 5,6 .

In what follows, we focus on the four-point correlators in the Lee-Yang series, i.e. the parameters $a_{i}=a_{1,-n_{i}}$ and $b=\sqrt{2 / p}, \mathcal{I}_{4}\left(n_{1}, n_{2}, n_{3}, n_{4}\right)=I_{4}\left(a_{1,-n_{1}}, a_{1,-n_{2}}, a_{1,-n_{3}}, a_{1,-n_{4}}\right)$.

$$
\mathcal{I}_{4}\left(n_{i}\right)=\int_{\mathcal{M}_{0,3}} d^{2} z\left\langle\mathbb{U}_{1, n_{1}}(z) \mathbb{W}_{1, n_{2}}(0) \mathbb{W}_{1, n_{3}}(1) \mathbb{W}_{1, n_{4}}(\infty)\right\rangle
$$

Taking into account the explicit form of the correlation functions in the ghost sector

$$
\langle C(0) C(1) C(\infty)\rangle=1,
$$

we obtain

$$
\begin{aligned}
\mathcal{I}_{4}\left(n_{i}\right)=\int_{\mathcal{M}_{0,3}} d^{2} z\left\langle\Phi_{1, n_{1}}(z) \Phi_{1, n_{2}}(0) \Phi_{1, n_{3}}(1) \Phi_{1, n_{4}}(\infty)\right\rangle \times \\
\times\left\langle V_{1,-n_{1}}(z) V_{1,-n_{2}}(0) V_{1,-n_{3}}(1) V_{1,-n_{4}}(\infty)\right\rangle .
\end{aligned}
$$

For further purposes this expression can be conveniently written in more explicit form, for details, see appendix A.

\section{Discrete terms}

In the moduli integral (3.12) the integrand contains four-point Liouville correlation function. Its computation involves integration in momentum $P$, as in (2.5). The integrand is a product of LFT structure constants (2.6)

$$
\mathbb{C}^{L}\left(a_{1}, a_{2}, p\right) \mathbb{C}^{L}\left(Q-p, a_{3}, a_{4}\right)
$$

and the conformal blocks. In the case, where $\Re\left(\left|Q / 2-a_{i}\right|\right)+\Re\left(\left|Q / 2-a_{j}\right|\right)<Q / 2$ for $i \neq j$ the contour of integration goes along the real axis. This corresponds to the fact that in this case the correlator is a sum over intermediate states in the Hilbert space of Liouville theory. When this condition is not satisfied, meromorphic continuation of the correlation functions is required. It can be achieved by deforming the integration contour (see, e.g. $[3,15]$ ). Basically, in this case poles of structure constants intersect the real line and one needs to add corresponding residues to the total integral, as depicted in figure 1 . These residues are called discrete terms. If, for example, $Q / 2-a_{i}>0$ then the corresponding poles come from zeros in

$$
\begin{aligned}
\Upsilon\left(a_{i}+a_{j}-p\right) & =\Upsilon\left(p+\left(Q / 2-a_{i}+Q / 2-a_{j}\right)\right), \\
\Upsilon\left(a_{i}+a_{j}+p-Q\right) & =\Upsilon\left(p-\left(Q / 2-a_{i}+Q / 2-a_{j}\right)\right) .
\end{aligned}
$$

In this case one can easily see that the corresponding residues are to be taken at

$$
p=\left(Q / 2-a_{i}+Q / 2-a_{j}\right)-r / b-s b, p>Q / 2
$$

and in the reflected positions $Q-p$ with the same residues.

We note that expression (4.1) in principle may have a second order pole if both of the structure constants have poles for the momentum $p$. In what follows we assume that this is not the case, then the residues are computed easily using quasiperiodicity of $\Upsilon$-function and the fact that $\Upsilon(\varepsilon)=\Upsilon(b) \varepsilon+O\left(\varepsilon^{2}\right)$. 


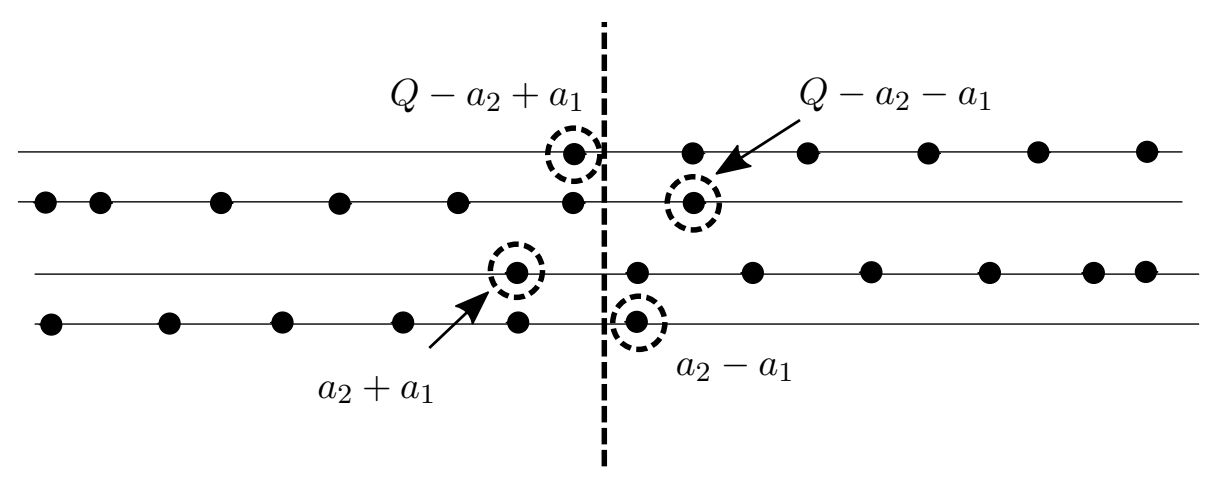

Figure 1. Poles of structure the constant and discrete terms.

\section{Generalization of HEM formula}

HEM formula (3.8), (3.9) was derived assuming that the fields labelled by 2, 3, 4 are constructed from generic non-degenerate matter fields. It was assumed that the formula is correct in more general case, where the number of conformal blocks in the matter sector is equal to $m n$.

In Minimal Models one can compute four-point function $\left\langle\Phi_{m, n} \Phi_{m_{2}, n_{2}} \Phi_{m_{3}, n_{3}} \Phi_{m_{4}, n_{4}}\right\rangle$ using operator product expansions $\Phi_{m, n} \Phi_{2}=\sum_{r, s}\left[\Phi_{r, s}\right]$ and $\Phi_{3} \Phi_{4}=\sum_{r^{\prime}, s^{\prime}}\left[\Phi_{r^{\prime}, s^{\prime}}\right]$, where the summation goes according to MM fusion rules (2.2). The holomorphic contribution of the pair $(r, s)$ in the intermediate channel defines a conformal block [5]. The number of terms in each OPE is not greater then the minimum of products of indices of the fields. Therefore the number of conformal blocks is not greater then the minimum of $m_{i} n_{i}$. For example, in the correlation function $\left\langle\Phi_{1,2} \Phi_{1,2} \Phi_{1,2} \Phi_{1,4}\right\rangle$ the only field, which can appear in the intermediate channel is $\Phi_{1,3}$ so that the number of conformal blocks is less then 2 . Thus the assumption, under which HEM formula was derived, is not satisfied. However, as we will see later, this formula is correct in more general case. The applicability condition is defined by both number of conformal blocks and the presence of discrete terms in the Liouville four-point function. In the example given above the old formula is correct for $\mathcal{M}(2 / 9)$, but is not correct for $\mathcal{M}(2 / 11)$.

Most of the consideration below is applicable to the general $\mathcal{M}\left(p^{\prime} / p\right)$ case, though we are going to use it for the Lee-Yang series. Our modification of the HEM formula reads:

$$
\Sigma_{\mathrm{MHEM}}=\Sigma_{\mathrm{HEM}}-\sum_{i=2}^{4} \sum_{(r, s) \in F_{i} \cap R_{i}} 2 \lambda_{r, s},
$$

where $F_{i}$ is the fusion set of $\Phi_{m, n} \Phi_{i}\left(\Phi_{m, n} \Phi_{i} \rightarrow \Phi_{r, s}\right)$ and $R_{i}$ is the set of discrete terms in the OPE of $V_{j} V_{k}\left(V_{j} V_{k} \rightarrow V_{r,-s}\right)$ and $\{i, j, k\}=\{2,3,4\}$.

Proof of the modified HEM formula. It appears that for Generalized Minimal Models correlation number of four degenerate fields is not well defined. To compute the correlator of degenerate fields we start with the correlator of one degenerate and three arbitrary 
nondegenerate fields $\mathbb{W}_{a_{i}}$. Then HEM formula reads:

$$
\left\langle\mathbb{U}_{a_{m_{1}, n_{1}}} \mathbb{W}_{a_{2}} \mathbb{W}_{a_{3}} \mathbb{W}_{a_{4}}\right\rangle=2 m n \lambda_{m, n}+\sum_{i=2}^{4} \sum_{r, s}^{(m, n)}\left(\left|\lambda_{i}-\lambda_{r, s}\right|_{\operatorname{Re}}-\lambda_{m, n}\right) .
$$

We are interested in the limit $a_{i} \rightarrow a_{m_{i}, n_{i}}$. The direct approach based on the conformal block decomposition gives the formula for the correlator (5.2):

$$
\begin{aligned}
\mathcal{I}_{4}\left(a_{m_{1}, n_{1}}, a_{i}\right)= & 2 \sum_{k} \mathbb{C}_{12}^{k} \mathbb{C}_{k 34} \\
\pi^{2} \int^{\prime} \frac{d P}{4 \pi} \mathbb{C}^{L}\left(a_{m_{1}, n_{1}}, a_{2}, Q / 2-i P\right) \mathbb{C}^{L}\left(Q / 2+i P, a_{3}, a_{4}\right) & \\
& \sum_{l} \sum_{j}\left(b_{j}(P) b_{l-j}(P) \Phi\left(\Delta^{L}(P)+\Delta_{k}^{M}-1, j, l-j\right)\right)+\circlearrowright,
\end{aligned}
$$

where $\circlearrowright$ denotes two other terms with cyclic permutations of $a_{2}, a_{3}, a_{4}$ (see appendix A for details). Let us focus on one of these terms. In (5.3) some Minimal Model structure constants $\mathbb{C}_{12}^{k}$ and $\mathbb{C}_{k 34}$ become zero in the desired limit. ${ }^{7}$ Let us denote the corresponding terms in (5.3) as

$$
\left\langle\mathbb{U}_{a_{m_{1}, n_{1}}} \mathbb{W}_{a_{m_{2}, n_{2}}} \mathbb{W}_{a_{m_{3}, n_{3}}} \mathbb{W}_{a_{m_{4}, n_{4}}}\right\rangle^{k}+\circlearrowright
$$

When the matter is represented by Minimal Models, these terms do not appear in the expression for the correlation numbers because of the fusion rules. However, sometimes these terms do not vanish automatically in the limit, so that to get an answer for Minimal Models we take the limit of (5.2) and then subtract terms (5.4)

$$
\begin{aligned}
& \left\langle\mathbb{U}_{a_{m_{1}, n_{1}}} \mathbb{W}_{a_{m_{2}, n_{2}}} \mathbb{W}_{a_{m_{3}, n_{3}}} \mathbb{W}_{a_{m_{4}, n_{4}}}\right\rangle= \\
& \lim _{a_{i} \rightarrow a_{m_{i}, n_{i}}}\left[\left\langle\mathbb{U}_{a_{m_{1}, n_{1}}} \mathbb{W}_{a_{2}} \mathbb{W}_{a_{3}} \mathbb{W}_{a_{4}}\right\rangle\right]-\left(\sum_{k}\left\langle\mathbb{U}_{a_{m_{1}, n_{1}}} \mathbb{W}_{a_{m_{2}, n_{2}}} \mathbb{W}_{a_{m_{3}, n_{3}}} \mathbb{W}_{a_{m_{4}, n_{4}}}\right\rangle^{k}+\circlearrowright\right)
\end{aligned}
$$

Let us compute the contribution of (5.4). Some of these terms do not vanish because $\Phi\left(\Delta^{L}(P)+\Delta_{k}^{M}-1, j, l-j\right)$, arising from the $x$-integration, has a pole and annihilates zero appearing in the structure constant (2.3). Explicitly one has (see (A.8))

$$
\Phi(A, r, l)=\frac{(16)^{2 A}}{\pi(2 A+r+l)} \int_{-1 / 2}^{1 / 2} \cos (\pi(r-l) x) e^{-\pi \sqrt{1-x^{2}}(2 A+r+l)} d x
$$

so that it has a pole when $2 A+r+l=0$ and $r-l$ is odd or zero. In our case it implies $r=l=0$ and $A=0$, which leads us to the conclusion that in the intermediate channel the Liouville dimension $\Delta^{L}(P)$ should be dressing for the matter dimension $\Delta_{k}^{M}$ in the sense that $\Delta^{L}(P)+\Delta_{k}^{M}=1$. This can be possible only if Liouville correlation function has specific discrete terms, i.e. $i P \rightarrow \lambda_{m,-n}$. The first thing to notice is that nonzero terms (5.4) appear precisely if $k=(r, s) \in F_{i} \cap R_{i}$ as in the formula (5.1).

\footnotetext{
${ }^{7}$ There are some complications when these structure constants do not vanish even if they should do according to the fusion rules [2], but this is not the case for Lee-Yang series and is not discussed here.
} 
Let us compute the value of each of these terms. We have

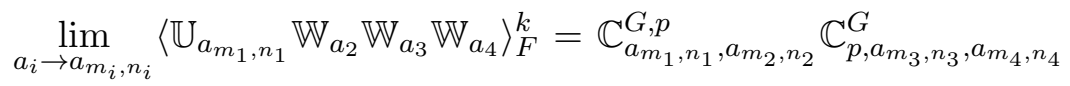

$$
\begin{aligned}
& =\mathbb{C}_{a_{m_{1}, n_{1}}, a_{m_{2}, n_{2}, p},}^{G}\left(D_{p, p}^{G}\right)^{-1} \mathbb{C}_{p, a_{m_{3}, n_{3}}, a_{m_{4}, n_{4}}}^{G} \\
& =2 \lambda_{k} \kappa \prod_{j=1}^{4} N\left(a_{m_{i}, n_{i}}\right) \text {, }
\end{aligned}
$$

where $D^{G}$ and $\mathbb{C}^{G}$ denote MLG two- and three-point functions, $\kappa$ is given in (3.10) and $\lambda_{k}$ is $Q / 2-a_{k}$. Taking (5.5), (5.6) into account one derives modified HEM formula (5.1).

Let us now accurately prove (5.6). We start from the formula (5.3). Taking residue in the discrete terms and using reflection relation in LFT, $\mathbb{C}^{L}\left(a_{m_{1}, n_{1}}, a_{2}, p_{k}\right) R_{L}\left(p_{k}\right)^{-1}=$ $\mathbb{C}^{L}\left(a_{m_{1}, n_{1}}, a_{2}, Q-p_{k}\right)$, we have

$$
\begin{aligned}
\left\langle\mathbb{U}_{a_{m_{1}, n_{1}}} \mathbb{W}_{a_{2}} \mathbb{W}_{a_{3}} \mathbb{W}_{a_{4}}\right\rangle_{F}^{k}= & 2 \mathbb{C}^{M}\left(\alpha_{m_{1}, n_{1}}, \alpha_{2}, \alpha_{k}\right) \mathbb{C}^{M}\left(\alpha_{k}, \alpha_{3}, \alpha_{4}\right) \\
& \pi^{2} \mathbb{C}^{L}\left(a_{m_{1}, n_{1}}, a_{2}, p_{k}\right) R_{L}\left(p_{k}\right)^{-1} \operatorname{Res}_{p \rightarrow p_{k}}\left[\mathbb{C}^{L}\left(p_{k}, a_{3}, a_{4}\right)\right] \\
& \sum_{l} \sum_{j}\left(b_{j}\left(p_{k}\right) b_{l-j}\left(p_{k}\right) \Phi\left(A_{k}\left(p_{k}\right), j, l-j\right)\right)+\circlearrowright .
\end{aligned}
$$

In the last formula we used the notations $p=Q / 2+i P, A_{k}(P)=\Delta_{a_{k}}^{L}+\Delta_{\alpha_{k}}^{M}-1$ and $p_{k}$ is the value of $p$ corresponding to the discrete term of interest. In (5.7) we also took into account two equivalent symmetric residues, which produces the factor of 2 . Now we denote $\varepsilon=p_{k}-a_{k}$, where $\Delta_{a_{k}}^{L}+\Delta_{\alpha_{k}}^{M}=1$ and $\Delta_{\alpha_{k}}^{M}$ is the dimension of the intermediate field in the MM conformal block. We ignore terms of order $o(\varepsilon)$ and multiply Minimal Model structure constants by Liouville ones to get MLG three-point functions. In this way we obtain

$$
\begin{aligned}
\left\langle\mathbb{U}_{a_{m_{1}, n_{1}}} \mathbb{W}_{a_{2}} \mathbb{W}_{a_{3}} \mathbb{W}_{a_{4}}\right\rangle_{\mathbf{F}}^{k} \sim & 2 \mathbb{C}^{M}\left(\alpha_{m_{1}, n_{1}}, \alpha_{2}, \alpha_{k}\right) \cdot \mathbb{C}^{L}\left(a_{m_{1}, n_{1}}, a_{2}, p_{k}\right) \\
& \mathbb{C}^{M}\left(\alpha_{k}, \alpha_{3}, \alpha_{4}\right) \cdot(-\varepsilon) \mathbb{C}^{L}\left(\alpha_{k}, a_{3}, a_{4}\right) \\
& \pi^{2} R_{L}\left(p_{k}\right)^{-1} \sum_{l} \sum_{j}\left(b_{j}\left(p_{k}\right) b_{l-j}\left(p_{k}\right) \Phi\left(A_{k}\left(p_{k}\right), j, l-j\right)\right)+\circlearrowright
\end{aligned}
$$

To compute this expression we note that $\Phi$ has a pole in $\varepsilon$ only if $j=l=0$, so that we can ignore other terms. Using the explicit formula for $\Phi$ we find

$$
\Phi\left(A_{k}\left(p_{k}\right), 0,0\right) \sim \frac{1}{2 \pi A_{k}\left(p_{k}\right)} \sim \frac{1}{2 \pi \Delta^{L}\left(p_{k}\right)^{\prime} \varepsilon} .
$$

Now we expand the value of $R_{L}\left(p_{k}\right)$ :

$$
R_{L}(a)=\left(\pi \mu \gamma\left(b^{2}\right)\right)^{(Q-2 a) / b} \frac{\gamma\left(2 a b-b^{2}\right)}{b^{2} \gamma\left(2-2 a b^{-1}+b^{-2}\right)}
$$

and two- and three-point functions in MLG are correspondingly:

$$
\begin{aligned}
D_{a, a}^{G} & =\frac{\kappa}{2 \lambda_{a}} N(a)^{2}, \\
\mathbb{C}_{a_{1}, a_{2}, a_{3}}^{G} & =b \kappa \prod_{i=1}^{3} N\left(a_{i}\right) .
\end{aligned}
$$

Using these expressions we finally arrive to the formula (5.6) and thus prove (5.1). 
For Lee-Yang series we can further simplify (5.1). Without loss of generality let $n_{1} \leq$ $n_{2} \leq n_{3} \leq n_{4} \leq s, p=2 s+1$. Then only the term

$$
\sum_{(1, s) \in R_{4}} 2 \lambda_{1, s}
$$

survives in the sum. If $\sum_{i} n_{i}$ is even, then the last expression is equal to

$$
\sum_{s=n_{2}+n_{3}+1: 2}^{\min \left(n_{1}+n_{4}-1, s\right)} 2 \lambda_{1, s}=\frac{1}{2 \sqrt{2 p}}\left(\hat{F}\left(\min \left(n_{1}+n_{4}, n_{2}+n_{3}\right)\right)-\hat{F}\left(n_{1}+n_{4}\right)\right)
$$

where $\hat{F}(n)=(s+1-n)(s-n) \theta(n \leq s)$. If $\sum_{i} n_{i}$ is odd, then (5.12) equals to

$$
\sum_{s=n_{2}+n_{3}+1: 2}^{s} 2 \lambda_{1, s}=\frac{1}{2 \sqrt{2 p}} \hat{F}\left(n_{2}+n_{3}\right)=\frac{1}{2 \sqrt{2 p}}\left(\hat{F}\left(\min \left(n_{1}+n_{4}, n_{2}+n_{3}\right)\right)-\hat{F}\left(n_{1}+n_{4}\right)\right),
$$

where the last equality is due to $n_{1}+n_{4}>s$ and $n_{2}+n_{3}<s$.

Now for Lee-Yang series we can rewrite (5.1) as

$$
\Sigma_{\mathrm{MHEM}}=\Sigma_{\mathrm{HEM}}-\frac{1}{2 \sqrt{2 p}}\left(\hat{F}\left(\min \left(n_{1}+n_{4}, n_{2}+n_{3}\right)\right)-\hat{F}\left(n_{1}+n_{4}\right)\right) .
$$

\section{Comparison with Douglas equation approach}

In this section we compare our results with the results of the Douglas equation approach.

Using identification $\Phi_{1, n}=\Phi_{1, p-n}$ in Lee-Yang series we will study fields $\mathbb{U}_{1, n}$ with $n \leq s$, where $p=2 s+1$. Our modified HEM approach gives formula (5.13). For comparison purposes we consider a normalization independent version of this formula:

$$
\begin{aligned}
\frac{\left\langle\left\langle\mathbb{U}_{m_{1}, n_{1}} \mathbb{U}_{m_{2}, n_{2}} \mathbb{U}_{m_{3}, n_{3}} \mathbb{U}_{m_{4}, n_{4}}\right\rangle\right\rangle}{\left(\prod_{i=1}^{4}\right.} & \left.\left\langle\left\langle\mathbb{U}_{m_{i}, n_{i}}^{2}\right\rangle\right\rangle\right)^{1 / 2} \\
= & \frac{\prod_{i=1}^{4}\left|m_{i} p-n_{i} p^{\prime}\right|^{1 / 2}}{2 p\left(p+p^{\prime}\right)\left(p-p^{\prime}\right)} \\
& \times\left(\sum_{i=2}^{4} \sum_{r=-\left(m_{1}-1\right)}^{m_{1}-1} \sum_{t=-\left(n_{1}-1\right)}^{n_{1}-1}\left|\left(m_{i}-r\right) p-\left(n_{i}-t\right) p^{\prime}\right|-m_{1} n_{1}\left(m_{1} p+n_{1} p^{\prime}\right)\right) \\
= & \frac{\prod_{i=1}^{4}\left|m_{i} p-n_{i} p^{\prime}\right|^{1 / 2}}{2 p\left(p+p^{\prime}\right)\left(p-p^{\prime}\right)}\left(-2 \sqrt{p p^{\prime}} \Sigma_{\operatorname{MHEM}}\left(m_{i}, n_{i}\right)\right),
\end{aligned}
$$

where $p^{\prime}=2$ and $n_{i}=1 .{ }^{8}$ We denote $\Sigma^{\prime}\left(m_{i}, n_{i}\right)=-2 \sqrt{p p^{\prime}} \Sigma\left(m_{i}, n_{i}\right)$ and expect it to be an integer number, so that in the comparison it will be the most convenient quantity.

The numerical quantity to be compared with $\Sigma_{\text {MHEM }}^{\prime}$ is

$$
\Sigma_{\mathrm{NUM}}^{\prime}\left(m_{i}, n_{i}\right)=-2 \sqrt{p p^{\prime}} \frac{I_{4}\left(m_{i}, n_{i}\right)}{\prod_{i=1}^{4} N\left(m_{i}, n_{i}\right) \kappa} .
$$

\footnotetext{
${ }^{8}$ We do not specify $p^{\prime}$ and $m_{i}$ in (6.1) in order to make the structure of this formula more clear.
} 
In the framework of the Douglas equation approach there are two formulae for the fourpoint correlation numbers. First of them $[9,12]$ after renormalization can be written as

$$
\begin{aligned}
\Sigma_{\mathrm{DSE}}^{\prime}\left(n_{i}\right)= & -\hat{F}(0)+\sum_{i=1}^{4} \hat{F}\left(n_{i}\right)-\hat{F}\left(\min \left(n_{1}+n_{2}, n_{3}+n_{4}\right)\right) \\
& -\hat{F}\left(\min \left(n_{1}+n_{3}, n_{2}+n_{4}\right)\right)-\hat{F}\left(\min \left(n_{1}+n_{4}, n_{3}+n_{2}\right)\right),
\end{aligned}
$$

where $\hat{F}(n)=(s+1-n)(s-n) \theta(n \leq s)$.

The second one is proposed in [13] and coincides with the above one when the number of conformal blocks is maximal and does not otherwise.

Proposition 6.1. The formula for four-point correlation numbers in Douglas equation approach is equivalent to the modified HEM formula:

$$
\Sigma_{\operatorname{MHEM}}\left(n_{i}\right)=\Sigma_{\mathrm{DSE}}\left(n_{i}\right) .
$$

Moreover, if there are no discrete terms in the operator product expansion $V_{1, n_{2}} V_{1, n_{3}}$, then we also have $\Sigma_{\text {HEM }}=\Sigma_{\text {MHEM }}$. The proof can be found in appendix B.

All our numerical computations of correlation numbers in various models confirm that the formula (5.13) is correct. In order to give some reference points we list some of the numerical results compared with Douglas equation approach and with the old HEM formula in tables 1, 2. In the tables correlator 12121214 means $\left\langle\mathbb{U}_{1,2} \mathbb{W}_{1,2} \mathbb{W}_{1,2} \mathbb{W}_{1,4}\right\rangle$ and so on. Sign * after the correlator means that there are discrete terms in Liouville OPE of any of the four fields, sign ${ }^{\dagger}$ means that there is a discrepancy between different approaches $\left(\Sigma_{N U M, D S E, H E M}^{\prime}\right.$ correspond to numerical computation, Douglas equation approach and higher equations of motion approach respectively).

In the table 1 we give some results on correlation numbers in different models. Note that in the table we also presented the results for the Minimal Model $\mathcal{M}(4 / 15)$, which does not belong to the Lee-Yang series. We list a larger set of correlation numbers in the table 2 for the model $\mathcal{M}(2 / 15)$.

\section{Discussion}

We have considered the direct approach to Liouville Minimal Gravity. Our main result is the formula (5.1) for four-point correlation numbers in the Lee-Yang series. This formula generalizes the old one (3.9) proposed in [3]. We show that our modified HEM formula is equivalent to the DSE formula (6.3). We also performed numerical checks, which confirm our results in the region of parameters where the old formula was not applicable.

Below we state some questions which naturally arise from the present considerations.

If the matter sector is represented by the Minimal Model with $p^{\prime}>2$, in Douglas equation approach it is impossible to fulfil all the Minimal Model fusion rules as was shown in $[8,9]$. So it would be interesting to see how does the correspondence between DSE and conformal field theory approaches extends to other Minimal Models.

In [2] there was obtained a formula for three-point functions in GMM. It coincides with the one obtained by Dotsenko and Fateev in [14] when it is not forbidden by fusion 


\begin{tabular}{|c|c|c|c|}
\hline$m_{i} n_{i}$ & $\left|\Sigma_{\mathrm{NUM}}^{\prime}\left(m_{i}, n_{i}\right)\right| / 2$ num. & $\Sigma_{\mathrm{DSE}}^{\prime}\left(m_{i}, n_{i}\right) / 2$ exact & $\Sigma_{\mathrm{HEM}}^{\prime}\left(m_{i}, n_{i}\right) / 2$ exact \\
\hline $2 / 9$ & - & - & - \\
\hline 12121212 & 2.00002 & 2 & 2 \\
\hline 13131212 & 2.00031 & -2 & -2 \\
\hline $1214 \quad 1212$ & 1.00003 & -1 & -1 \\
\hline 13121313 & 4.00016 & -4 & -4 \\
\hline $2 / 11$ & - & - & - \\
\hline 13151313 & 5.99976 & -6 & -6 \\
\hline $12141212^{* \dagger}$ & 1.000001 & 1 & 2 \\
\hline $2 / 13$ & 一 & 一 & 一 \\
\hline $12141212^{* \dagger}$ & 3.0001 & 3 & 6 \\
\hline $4 / 15$ & - & - & - \\
\hline 13171313 & 2.00009 & $\mathrm{~N} / \mathrm{A}$ & -2 \\
\hline 13151313 & 10.9998 & $\mathrm{~N} / \mathrm{A}$ & -11 \\
\hline
\end{tabular}

Table 1. Numerical data for $\Sigma^{\prime} . *-$ means discrete terms. ${ }^{\dagger}-$ discrepancies.

rules. But for some reason this formula gives a nonzero result for some structure constants which should vanish according to the fusion rules. Taking into account this fact would clearly lead to further complications for four-point correlation numbers in general Minimal Models, as mentioned in section 5. In [2] the prescription to obtain MM from GMM is to multiply the GMM structure constants by fusion algebra constants. As far as we know, there is no good understanding of this phenomenon, but it can also be connected with the previous question and with the fusion rules problem in MLG. For instance, without this additional restriction MLG three-point functions are always nonzero, that requires a better understanding. Some insight to this problem can be found in [28], where Liouville theory with $c \leq 1$ (GMM in our language) is discussed.

\section{Acknowledgments}

We thank Alexander Belavin for useful discussions and all other people with whom authors discussed the subject. The work was performed with financial support from the Russian Science Foundation (Grant No.14-12-01383). V.B. thanks G. Mussardo and INFN for possibility to visit SISSA, where the final part of this work was performed.

\section{A Conformal block decomposition in correlation numbers}

In this appendix we derive convenient representation for the correlation numbers.

The following considerations are known in the literature, see e.g. [29]. We start from the formula (3.12) for the correlation numbers and use the symmetry of the integrals under modular transformations in order to reduce the integration from the whole complex plane to the fundamental domain. The modular subgroup of projective transformations divides the complex plane into six regions. The fundamental region is defined as $\mathbf{G}=\{\operatorname{Re} x<$ 


\begin{tabular}{|c|c|c|c|}
\hline$m_{i} n_{i}$ & $\left|\Sigma_{\text {NUM }}^{\prime}\left(m_{i}, n_{i}\right)\right| / 2$ num. & $\sum_{\mathrm{DSE}}^{\prime}\left(m_{i}, n_{i}\right) / 2$ exact & $\sum_{\text {HEM }}^{\prime}\left(m_{i}, n_{i}\right) / 2$ exact \\
\hline $2 / 15$ & - & - & - \\
\hline $12121315^{* \dagger}$ & 3.08 & 3 & 6 \\
\hline $12121416^{* \dagger}$ & 1.025 & 1 & 2 \\
\hline $12121515^{*}$ & 1.98 & 2 & 2 \\
\hline $12121517^{*}$ & 1.03 & -1 & -1 \\
\hline $12121616^{*}$ & 2.06 & -2 & -2 \\
\hline $12121717^{*}$ & 4.09 & -4 & -4 \\
\hline $12131316^{* \dagger}$ & 1.01 & 1 & 2 \\
\hline $12131415^{*}$ & 1.99 & 2 & 2 \\
\hline $12131417^{*}$ & 1.015 & 1 & 1 \\
\hline $12131516^{*}$ & 2.03 & -2 & -2 \\
\hline $12131617^{*}$ & 5.07 & -5 & -5 \\
\hline $12141414^{*}$ & 1.995 & 2 & 2 \\
\hline $12141416^{*}$ & 2.02 & -2 & -2 \\
\hline $12141515^{*}$ & 2.01 & -2 & -2 \\
\hline $12141517^{*}$ & 5.04 & -5 & -5 \\
\hline $12141616^{*}$ & 6.05 & -6 & -6 \\
\hline $12141717^{*}$ & 8.04 & -8 & -8 \\
\hline $13131315^{* \dagger}$ & 1.995 & 2 & 3 \\
\hline $13131317^{* \dagger}$ & 0.999 & -1 & 0 \\
\hline $13131414^{*}$ & 2.93 & 3 & 3 \\
\hline $13131416^{*}$ & 2.01 & -2 & -2 \\
\hline $13131515^{*}$ & 3.03 & -3 & -3 \\
\hline $13131517^{*}$ & 6.05 & -6 & -6 \\
\hline $13131616^{*}$ & 7.04 & -7 & -7 \\
\hline $13131717^{*}$ & 9.05 & -9 & -9 \\
\hline
\end{tabular}

Table 2. Numerical data for $\Sigma^{\prime} . *$ means discrete terms. ${ }^{\dagger}$ - discrepancy.

$1 / 2 ;|1-x|<1\}$. The other five regions are mapped to the fundamental one using one of the transformations $\mathcal{A}, \mathcal{B}, \mathcal{A B}, \mathcal{B} \mathcal{A}, \mathcal{A B} \mathcal{A}$, where $\mathcal{A}: z \rightarrow 1 / z$ and $\mathcal{B}: z \rightarrow 1-z$. Combining the projective transformations of the fields and the corresponding change of the variables in the integrals, we reduce the integration to the fundamental region. We note that the Jacobian of the transformation exactly cancels the transformation of the fields because their total conformal dimension is 1 . Then,

$$
\begin{array}{r}
\mathcal{I}_{4}\left(n_{i}\right)=2 \int_{\mathbf{G}} d^{2} z\left(\left\langle\mathbb{W}_{1}(0) \mathbb{U}_{2}(z) \mathbb{W}_{3}(1) \mathbb{W}_{4}(\infty)\right\rangle+\left\langle\mathbb{W}_{3}(0) \mathbb{U}_{2}(z) \mathbb{W}_{1}(1) \mathbb{W}_{4}(\infty)\right\rangle\right. \\
\left.+\left\langle\mathbb{W}_{4}(0) \mathbb{U}_{2}(z) \mathbb{W}_{3}(1) \mathbb{W}_{1}(\infty)\right\rangle\right)
\end{array}
$$

where the factor 2 in front counts the equivalent projective images (the order of the last two fields is not relevant) and $\mathbb{U}_{i}, \mathbb{W}_{i}$ stand for $\mathbb{U}_{a_{i}}, \mathbb{W}_{a_{i}}$. 
Conformal block decomposition. For a while, we omit some arguments that are easily reconstructed in the final expressions. In the matter sector,

$$
\begin{aligned}
\left\langle\Phi_{1}(0) \Phi_{2}(z) \Phi_{3}(1) \Phi_{4}(\infty)\right\rangle & =\sum_{k} c_{k}^{(1)}\left|\mathcal{F}_{k}^{(1)}(z)\right|^{2} \\
\left\langle\Phi_{3}(0) \Phi_{2}(z) \Phi_{1}(1) \Phi_{4}(\infty)\right\rangle & =\sum_{k} c_{k}^{(1)}\left|\mathcal{F}_{k}^{(3)}(z)\right|^{2} \\
\left\langle\Phi_{4}(0) \Phi_{2}(z) \Phi_{3}(1) \Phi_{1}(\infty)\right\rangle & =\sum_{k} c_{k}^{(1)}\left|\mathcal{F}_{k}^{(4)}(z)\right|^{2}
\end{aligned}
$$

Here the index $k$ corresponds to the channels in the degenerate OPE of the fields $\Phi_{i}$ and the coefficients $c_{k}$ are related to the basic structure constants $[2,14]$ :

$$
c_{k}^{(1)}=\mathbb{C}_{12}^{k} \mathbb{C}_{34}^{k}, c_{k}^{(3)}=\mathbb{C}_{32}^{k} \mathbb{C}_{14}^{k}, c_{k}^{(4)}=\mathbb{C}_{42}^{k} \mathbb{C}_{31}^{k} .
$$

In (A.2), $\mathcal{F}_{k}^{(i)}$ denotes the conformal blocks appearing in the $k$-channel for the given correlation function. In the Liouville sector we have

$$
\begin{aligned}
\left\langle V_{1}(z) V_{2}(0) V_{3}(1) V_{4}(\infty)\right\rangle & =\mathcal{R} \int^{\prime} \frac{d P}{4 \pi} r^{(1)}(P)\left|\mathcal{F}^{(1)}(P, z)\right|^{2} \\
\left\langle V_{3}(z) V_{2}(0) V_{1}(1) V_{4}(\infty)\right\rangle & =\mathcal{R} \int^{\prime} \frac{d P}{4 \pi} r^{(3)}(P)\left|\mathcal{F}^{(3)}(P, z)\right|^{2} \\
\left\langle V_{4}(z) V_{2}(0) V_{3}(1) V_{1}(\infty)\right\rangle & =\mathcal{R} \int^{\prime} \frac{d P}{4 \pi} r^{(4)}(P)\left|\mathcal{F}^{(4)}(P, z)\right|^{2}
\end{aligned}
$$

where

$$
\mathcal{R} r^{(1)}(P)=\mathbb{C}^{L}\left(a_{1}, a_{2}, Q / 2+i P\right) \mathbb{C}^{L}\left(Q / 2-i P, a_{3}, a_{4}\right)
$$

and so on. Here $\mathcal{R}$ stands for the momentum independent part of the product. In what follows we omit upper subscripts pointing permutations of the fields and summation with respect to them in the correlators.

The modular integral. It is efficient [30] to go to the universal cover of the moduli space $\mathcal{M}_{0,3}=S^{2} \backslash\{0,1, \infty\}$, that is to use elliptic transformation in the integration. We use the map

$$
\tau=i \frac{K(1-z)}{K(z)}
$$

where $K(z)$ is the complete elliptic integral of the first kind

$$
K(z)=\frac{1}{2} \int_{0}^{1} \frac{d t}{y}
$$

and $y^{2}=t(1-t)(1-z t)$. It can be verified that

$$
d z=\pi z(1-z) \theta_{3}^{4}(q) d \tau
$$

where the elliptic nome parameter

$$
q=e^{i \pi \tau}
$$


and theta constant

$$
\theta_{3}(q)=\sum_{n=-\infty}^{\infty} q^{n^{2}}
$$

Following [30] we can write

$$
\mathcal{F}\left(\Delta_{i}, \Delta \mid q\right)=(16 q)^{\Delta_{p}-\Delta_{0}} z^{\Delta_{0}-\Delta_{1}-\Delta_{2}}(1-z)^{\Delta_{0}-\Delta_{2}-\Delta_{3}} \theta_{3}^{12 \Delta_{0}-4 \sum \Delta_{i}}(q) H\left(\Delta_{i}, \Delta \mid q\right),
$$

in order to represent integral (A.1) in the following form

$$
\mathcal{I}_{4}\left(a_{i}\right)=2 \sum_{i}^{1,3,4} \pi^{2} \mathcal{R} \int^{\prime} \frac{d P}{4 \pi} \sum_{k} r^{(i)}(P) c_{k}^{(i)} \int_{\mathbf{F}}\left|16 q^{A_{k}(P)} H_{k}^{(i)}\left(q \mid \Delta_{p}^{L}\right) H_{k}^{(i)}(q)\right|^{2} \mathrm{~d}^{2} \tau,
$$

where $\mathbf{F}=\{|\tau|>1 ;|\operatorname{Re} \tau|<1 / 2\}, A_{k}(P)=\Delta^{L}(P)+\Delta_{k}^{M}-1$ is sum of conformal dimensions in the intermediate channel minus 1 and $H\left(\Delta_{i}, \Delta \mid q\right)$ is a series in $q$ of the form $1+O(q)$, which is computed using recurrence relation [30].

Numerics. With (A.5), the calculation reduces to the numerical integration of several integrals of the general form

$$
\int_{\mathbf{F}}\left|z(1-z) \theta_{3}^{4}(q) \mathcal{F}_{P}(z)\right|^{2} d^{2} \tau
$$

where $\mathcal{F}_{P}(z)$ is some Liouville conformal block like in (A.2) or some more complicated composite expression like in (A.3). The integrand can be developed as a power series in $q$ according to

$$
z(1-z) \theta_{3}^{4}(q) \mathcal{F}_{P}(z)=(16 q)^{\alpha} \sum_{r=0}^{\infty} b_{r}(P) q^{r}
$$

and the same for $\bar{q}$. In each term, we can integrate in $\tau_{2}=\operatorname{Im} \tau$ explicitly with the result conviniently represented in terms of the function

$$
\Phi(A, r, l)=\int_{\mathbf{F}} d^{2} \tau|16 q|^{2 A} q^{r} \bar{q}^{l}=\frac{(16)^{2 A}}{\pi(2 A+r+l)} \int_{-1 / 2}^{1 / 2} \cos (\pi(r-l) x) e^{-\pi \sqrt{1-x^{2}}(2 A+r+l)} d x .
$$

Using explicit formulae (A.5), (A.7), (A.8) we finally obtain the following expression for (A.5):

$$
\begin{aligned}
\mathcal{I}_{4}\left(a_{i}\right)= & \\
& 2 \sum_{i}^{1,3,4} \sum_{k} c_{k}^{(i)} \pi^{2} \mathcal{R} \int^{\prime} \frac{d P}{4 \pi} r^{(i)}(P) \sum_{l} \sum_{j}\left(b_{j}(P) b_{l-j}(P) \Phi\left(A_{k}(P), j, l-j\right)\right),
\end{aligned}
$$

where $b_{j}(P)=\left[q^{j}\right]\left(H^{(i)}\left(q \mid \Delta_{p}^{L}\right) H_{k}^{(i)}(q)\right)$ is a $q^{j}$ th term in the expansion of the elliptic conformal blocks and $A_{k}(P)=\Delta^{L}(P)+\Delta_{k}^{M}-1$ as above. Each term in (A.9) is suppressed by a factor $\max _{\mathbf{F}}|q|^{2 l}$ and the series in $l$ converges very rapidly.

Main source of numerical errors in these computations is a method of computing product of Liouville structure constants, namely functions $r^{(i)}(P)$. 


\section{B Proof of the proposition 6.1}

Here we prove that

$$
\Sigma_{\mathrm{MHEM}}^{\prime}\left(n_{i}\right)=\Sigma_{\mathrm{DSE}}^{\prime}\left(n_{i}\right) .
$$

Let $n_{1} \leq n_{2} \leq n_{3} \leq n_{4} \leq s$. For our purposes we write $\Sigma_{\mathrm{DSE}}^{\prime}$ and $\Sigma_{\mathrm{MHEM}}^{\prime}$ as

$$
\Sigma_{\mathrm{DSE}}^{\prime}=-\hat{F}(0)+\sum_{i=1}^{4} \hat{F}\left(n_{i}\right)-\hat{F}\left(n_{1}+n_{2}\right)-\hat{F}\left(n_{1}+n_{3}\right)-\hat{F}\left(\min \left(n_{1}+n_{4}, n_{2}+n_{3}\right)\right)
$$

and

$$
\Sigma_{\text {MHEM }}^{\prime}=\sum_{i=2}^{4} \sum_{t}^{\left(n_{1}\right)}\left|p-2\left(n_{i}+t\right)\right|-n_{1}\left(p+2 n_{1}\right)+-\hat{F}\left(\min \left(n_{1}+n_{4}, n_{2}+n_{3}\right)\right)+\hat{F}\left(n_{1}+n_{4}\right) .
$$

First, we need to show that the old formula $\Sigma_{\text {HEM }}^{\prime}$ coincides with $\Sigma_{\text {DSE }}^{*}$, where we have introduced

$$
\Sigma_{\mathrm{DSE}}^{*}=-\hat{F}(0)+\sum_{i=1}^{4} \hat{F}\left(n_{i}\right)-\hat{F}\left(n_{1}+n_{2}\right)-\hat{F}\left(n_{1}+n_{3}\right)-\hat{F}\left(n_{1}+n_{4}\right) .
$$

If $n_{1}+n_{i} \leq s+1$, in $\Sigma_{\mathrm{HEM}}^{\prime}$ all expressions under modules are positive and in $\Sigma_{\mathrm{DSE}}^{\prime}$ all $\hat{F}$ are equal to $\hat{F}_{0}$, so that both $\Sigma^{\prime}$ simplify to

$$
2 n_{1}\left(p-\sum_{i} n_{i}\right)
$$

When $n_{1}+n_{i} \geq s+2$ for some $i, \Sigma_{\text {HEM }}^{\prime}$ gets a correction to (B.5) because of the modules equal to

$$
-2 \sum_{t: p-2\left(n_{i}+t\right)<0}\left(p-2\left(n_{i}+t\right)\right)=\left(s+1-n_{1}-n_{i}\right)\left(s-n_{1}-n_{i}\right) .
$$

$\Sigma_{\text {DSE }}^{*}$ gets a correction because of the Heaviside theta function equal to $\hat{F}\left(n_{1}+n_{i}\right)=$ $\left(s+1-n_{1}-n_{i}\right)\left(s-n_{1}-n_{i}\right)$, which finishes the proof.

Now the initial statement (B.1) follows immediately from the definitions.

Open Access. This article is distributed under the terms of the Creative Commons Attribution License (CC-BY 4.0), which permits any use, distribution and reproduction in any medium, provided the original author(s) and source are credited.

\section{References}

[1] A. Polyakov, Quantum geometry of fermionic strings, Phys. Lett. B 103 (1981) 211.

[2] A.B. Zamolodchikov, Three-point function in the minimal Liouville gravity, Theor. Math. Phys. 142 (2005) 183 [hep-th/0505063] [INSPIRE].

[3] A.A. Belavin and A.B. Zamolodchikov, Integrals over moduli spaces, ground ring and four-point function in minimal Liouville gravity, Theor. Math. Phys. 147 (2006) 729 [INSPIRE]. 
[4] Al. Zamolodchikov, Higher equations of motion in liouville field theory, Int. J. Mod. Phys. A 19 (2004) 510.

[5] A.A. Belavin, A.M. Polyakov and A.B. Zamolodchikov, Infinite conformal symmetry in two-dimensional quantum field theory, Nucl. Phys. B 241 (1984) 333 [InSPIRE].

[6] P.H. Ginsparg and G.W. Moore, Lectures on $2 D$ gravity and $2 D$ string theory, hep-th/9304011 [INSPIRE].

[7] A.A. Belavin and V.A. Belavin, Frobenius manifolds, integrable hierarchies and minimal liouville gravity, JHEP 09 (2014) 151 [arXiv:1406.6661] [INSPIRE].

[8] V. Belavin, Unitary minimal Liouville gravity and Frobenius manifolds, JHEP 07 (2014) 129 [arXiv: 1405.4468] [INSPIRE].

[9] A. Belavin, B. Dubrovin and B. Mukhametzhanov, Minimal Liouville gravity correlation numbers from Douglas string equation, JHEP 01 (2014) 156 [arXiv:1310.5659] [INSPIRE].

[10] V.G. Knizhnik, A.M. Polyakov and A.B. Zamolodchikov, Fractal structure of $2 D$ quantum gravity, Mod. Phys. Lett. A 3 (1988) 819 [InSPIRE].

[11] G.W. Moore, N. Seiberg and M. Staudacher, From loops to states in 2D quantum gravity, Nucl. Phys. B 362 (1991) 665 [inSPIRE].

[12] A.A. Belavin and A.B. Zamolodchikov, On correlation numbers in $2 D$ minimal gravity and matrix models, J. Phys. A 42 (2009) 304004 [arXiv:0811.0450] [INSPIRE].

[13] V. Belavin and Yu. Rud, Matrix model approach to minimal Liouville gravity revisited, J. Phys. A 48 (2015) 18FT01 [arXiv:1502.05575] [INSPIRE].

[14] V. Dotsenko and V. Fateev, Four-point correlation functions and the operator algebra in $2 D$ conformal invariant theories with central charge $c \leqslant 1$, Nucl. Phys. B 251 (1985) 691.

[15] J. Teschner, Liouville theory revisited, Class. Quant. Grav. 18 (2001) R153 [hep-th/0104158] [INSPIRE].

[16] V. Kac., Infinite-dimensional Lie algebras, Progress in Mathematics volume 44, Springer, Germany (1984).

[17] H. Dorn and H.J. Otto, Two and three point functions in Liouville theory, Nucl. Phys. B 429 (1994) 375 [hep-th/9403141] [INSPIRE].

[18] A.B. Zamolodchikov and A.B. Zamolodchikov, Structure constants and conformal bootstrap in Liouville field theory, Nucl. Phys. B 477 (1996) 577 [hep-th/9506136] [INSPIRE].

[19] J. Teschner, On the Liouville three point function, Phys. Lett. B 363 (1995) 65 [hep-th/9507109] [INSPIRE].

[20] H. Dorn and H.J. Otto, On correlation functions for noncritical strings with $c<1$ but $d>1$, Phys. Lett. B 291 (1992) 39 [hep-th/9206053] [INSPIRE].

[21] F. David, Conformal field theories coupled to 2D gravity in the conformal gauge, Mod. Phys. Lett. A 3 (1988) 1651 [INSPIRE].

[22] J. Distler and H. Kawai, Conformal field theory and 2D quantum gravity or who's afraid of Joseph Liouville?, Nucl.Phys. B 231 (1989) 509.

[23] J. Polchinski, String theory, Cambridge University Press, Cambridge U.K. (1998). 
[24] E. Verlinde and H. Verlinde, Lectures on string perturbation theory, in the proceedings of the Trieste Spring School and Workshop on Superstrings (SUPERSTRINGS'88), April 11-22, Trieste, Italy (1988).

[25] D. Friedan, A tentative theory of large distance physics, JHEP 10 (2003) 063 [hep-th/0204131] [INSPIRE].

[26] I.R. Klebanov and A.M. Polyakov, Interaction of discrete states in two-dimensional string theory, Mod. Phys. Lett. A 6 (1991) 3273 [hep-th/9109032] [INSPIRE].

[27] E. Witten, Ground ring of two-dimensional string theory, Nucl. Phys. B 373 (1992) 187 [hep-th/9108004] [INSPIRE].

[28] S. Ribault and R. Santachiara, Liouville theory with a central charge less than one, JHEP 08 (2015) 109 [arXiv: 1503.02067] [INSPIRE].

[29] A. Zamolodchikov, Gravitational Yang-Lee model: four point function, Theor. Math. Phys. 151 (2007) 439 [hep-th/0604158] [INSPIRE].

[30] Al.B. Zamolodchikov, Conformal symmetry in two-dimensional space: Recursion representation of conformal block, Theor. Math. Phys. 73 (1987) 103. 